Nationale Rechtsordnungen 


\title{
Die Beamtenversorgung in der Bundesrepublik Deutschland
}

\author{
Yasemin Körtek
}

I. Einleitung

II. Beamte und Beamtenverhältnis

1. Verfassungsrechtlicher Hintergrund
a) Hergebrachte Grundsätze des Berufsbeamtentums

b) Alimentationsprinzip

2. Beurteilungsspielraum des Gesetzgebers bei der Gestaltung der Beamtenversorgung

III. Einfachgesetzliche Regelung der Beamtenversorgung

1. Verteilung der Gesetzgebungskompetenzen zwischen Bund und Ländern

2. Reformen
a) Versorgungsreformgesetz 1998
b) Versorgungsänderungsgesetz 2001
c) Neuordnung des Dienstrechts

3. Gesetzliche Bestimmung des Ruhegehalts
a) Anspruch auf Ruhegehalt und Höhe des Ruhegehalts
b) Mindestversorgung
c) Besteuerung von Versorgungsbezügen

IV. Bewertung 


\section{Einleitung}

Die soziale Sicherung von Beamten im Alter und bei Dienstunfähigkeit sowie ihrer Hinterbliebenen ist als Beamtenversorgung Teil des Beamtenrechts und stellt ein Sondersystem zur Alters- und Hinterbliebenensicherung neben der gesetzlichen Rentenversicherung dar. ${ }^{1}$ Die Beamtenversorgung dient wie die gesetzliche Rentenversicherung primär der finanziellen Absicherung bestimmter sozialer Risiken, unterscheidet sich aber in wesentlichen Punkten von der gesetzlichen Rentenversicherung.

Die gesetzliche Rentenversicherung ist ein auf Versicherungs- und Beitragspflicht beruhendes Vorsorgesystem, das im Umlageverfahren finanziert wird (§ 153 SGB VI). Die Versicherungspflicht in der gesetzlichen Rentenversicherung knüpft an eine Erwerbstätigkeit an. Kraft Gesetzes versicherungspflichtig sind in erster Linie Personen, die gegen Arbeitsentgelt oder zu ihrer Berufsausbildung beschäftigt werden ( $§ 1$ Abs. 1 S. 1 Nr. 1 SGB VI). ${ }^{2}$ Für bestimmte Personengruppen, die an sich versicherungspflichtig sind, besteht Versicherungsfreiheit kraft Gesetzes (§5 SGB VI) oder auf Antrag (§ 6 SGB VI). Beamte sind in der gesetzlichen Rentenversicherung versicherungsfrei ( $\$ 5$ Abs. 1 S. 1 Nr. 1 SGB VI). ${ }^{3}$ Im Rahmen eines abhängigen Beschäftigungsverhältnisses werden die Beiträge zur gesetzlichen Rentenversicherung von dem Versicherten und dem Arbeitgeber je zur Hälfte getragen ( $\$ 168$ Abs. 1 Ziff. 1 SGB VI) ${ }^{4}$ Beitragspflichtig ist Arbeitseinkommen bis zur Beitragsbemessungsgrenze, die entsprechend der Entwicklung der durchschnittlichen Bruttoarbeitsentgelte der Versicherten zum 1. Januar eines jeden Jahres geändert wird (§§ 159, 275a SGB VI). ${ }^{5}$ Das Ziel der gesetzlichen Rentenversicherung besteht vorrangig darin, dem Versicherten als Rentner den Lebensstandard zu sichern, den der Versicherte im Durchschnitt seines Arbeitslebens gehabt

1 Ruland, Die Beamtenversicherung, in: ders./Rürup (Hrsg.), Alterssicherung und Besteuerung, 2008, $\S 4$ Rdnr. 1, 2.

2 Personen, die nicht versicherungspflichtig sind, können sich in der gesetzlichen Rentenversicherung freiwillig versichern (§ 7 SGB VI).

3 Versicherungsfreiheit besteht des Weiteren z.B. für geringfügig Beschäftigte, die nicht schriftlich gegenüber ihrem Arbeitgeber auf die Versicherungsfreiheit verzichtet haben (§ 5 Abs. 2 SGB VI). Seit dem 1.4.1999 entrichten Arbeitgeber einen Pauschalbeitrag zur gesetzlichen Rentenversicherung, derzeit i.H.v. $15 \%$ (§ 172 Abs. 3 S. 1 SGB VI).

4 Der Beitragssatz für die gesetzliche Rentenversicherung beträgt 19,9 \% (2009). Die Arbeitgeberbeiträge sind steuerfrei. Arbeitnehmerbeiträge sind zwar steuerpflichtig, sind aber als Vorsorgeaufwendungen in bestimmten Umfang als Sonderausgaben steuerlich absetzbar ( $\S 3 \mathrm{Nr} .62,10 \mathrm{Abs} .1$ Nr. 2 EStG). Neben den Beiträgen der Versicherten und ihrer Arbeitgeber gehört der Bundeszuschuß zu den Einnahmen der gesetzlichen Rentenversicherung, den der Bund zu den Ausgaben der Rentenversicherung aus Steuermitteln leistet (§ 213 SGB VI).

5 Die Beitragsbemessungsgrenze liegt für 2009 in den alten Bundesländern bei 64.800 EUR und in den neuen Bundesländern bei 54.600 EUR. 
hat. ${ }^{6}$ Der Rente kommt eine Einkommensersatzfunktion zu. ${ }^{7}$ Dabei bietet die gesetzliche Rente eine Grundversorgung, die durch Zusatzversorgungen (z.B. betriebliche Altersversorgung) ergänzt wird. ${ }^{8}$ Die Beamtenversorgung dagegen umfaßt als Gesamtversorgung sowohl die Grund- als auch Zusatzversorgung der Beamten im Ruhestand. ${ }^{9}$ Sie wird daher als bifunktionales Sicherungssystem verstanden, dessen Finanzierung aus Haushaltsmitteln des Dienstherrn erfolgt und die Beamten zumindest keine „offenen“ Beiträge entrichten. ${ }^{10}$ Die verfassungsrechtliche Stellung des Berufsbeamtentums bedingt die besondere Behandlung von Beamten betreffend die Versorgung im Alter, bei Dienstunfähigkeit und der Hinterbliebenensicherung als Ausdruck der besonderen Fürsorgepflichten des Staates als Arbeitgeber der Beamten. ${ }^{11}$

Arbeiter und Angestellte des öffentlichen Dienstes, auf deren Rechtsverhältnisse die allgemeinen Regeln des Arbeitsrechts, modifiziert durch Tarifverträge, Anwendung finden, sind in der gesetzlichen Rentenversicherung pflichtversichert. Mit der Zusatzversorgung im öffentlichen Dienst über die Versorgungseinrichtungen des Bundes, der Länder und Gemeinden verfügt dieser Personenkreis über eine betriebliche Altersversorgung: Rentenleistungen werden neben der gesetzlichen Rentenversicherung auch von einer Versorgungseinrichtung, z.B. der Versorgungsanstalt des Bundes und der Länder, bezogen. Bisher war die Zusatzversorgung im öffentlichen Dienst wie die Beamtenversorgung auf eine Gesamtversorgung ausgerichtet; so wurde die Zusatzrente in Anleh-

6 Kolb, Gesetzliche Rentenversicherung, in: Sachverständigenkommission Alterssicherungssysteme, Berichtsband 2, 1983, S. 35; Ruland, Die Grundprinzipien des Rentenversicherungsrechts, in: ders. (Hrsg.), Handbuch der gesetzlichen Rentenversicherung, 1990, V 19 Rdnr. 90.

Die Höhe der Rente richtet sich vor allem nach der Höhe der während des Versicherungslebens durch Beiträge versicherten Arbeitsentgelte und Arbeitseinkommen, wobei das in den einzelnen Kalenderjahren durch Beiträge versicherte Arbeitsentgelt und Arbeitseinkommen in Entgeltpunkte umgerechnet wird (vgl. § 63 Abs. 1, 2 SGB VI).

7 BVerfGE 48, 346, 357; BSGE 30, 192, 199; Sodan, Verfassungsrechtliche Determinanten der gesetzlichen Rentenversicherung, NZS 2005, S. 561, 565.

8 Battis, Einführung, in: Alterssicherungssysteme im Vergleich, Schriften des WIÖD, Band 8, 1988, S. 7; Ruland, Rentenversicherung, in: von Maydell/Ruland/Becker (Hrsg.), Sozialrechtshandbuch, 4. Aufl. 2008, § 17 Rdnr. 13.

9 BVerfGE 114, 258, 294. Ausführlich Ruland, Möglichkeiten und Grenzen einer Annäherung der Beamtenversorgung an die gesetzliche Rentenversicherung, in: Sachverständigenkommission Alterssicherungssysteme, Anlageband B zum Gutachten, 1983.

10 Ruland, in: ders./Rürup (Hrsg.), Alterssicherung und Besteuerung (Fußn. 1), § 4 Rdnr. 4, 5, 59; Zacher (Mager), Bundesrepublik Deutschland, in: Zacher (Hrsg.), Alterssicherung im Rechtsvergleich, 1991, S. 170.

11 In der Folge ist die Abschaffung der Beamtenversorgung als Sondersystem sozialer Sicherung und eine etwaige Aufnahme der Beamtenversorgung in die gesetzliche Rentenversicherung nach geltendem Verfassungsrecht ausgeschlossen. Vgl. BVerfGE 44, 249, 269 f.; BVerfGE 76, 256, 319 f.; Petrasch, Die Alterssicherung der Beamten, Zugleich eine nähere Betrachtung des $\S 14$ a Bundesbesoldungsgesetz, 1999, S. 43, 52. 
nung an die Beamtenversorgung endgehaltbezogen berechnet. ${ }^{12}$ Doch hat die Zusatzversorgung im öffentlichen Dienst einen Systemwechsel erfahren: ${ }^{13}$ Ab dem 1.1.2002 ist an die Stelle der umlagenfinanzierten Zusatzversorgung die kapitalgedeckte Betriebsrente getreten. Die Betriebsrente wird nach einem Versorgungspunktemodell ermittelt. ${ }^{14}$

\section{Beamte und Beamtenverhältnis}

Im statusrechtlichen Sinn ist Beamter derjenige, der in einem öffentlich-rechtlichen Dienst- und Treueverhältnis steht, in das er durch Wahrung der gesetzlich vorgeschriebenen Form berufen worden ist. 15

Das Beamtenverhältnis als öffentlich-rechtliches Dienst- und Treueverhältnis 16 beruht auf hergebrachten Grundsätzen des Berufsbeamtentums. ${ }^{17}$

12 Gottwald, Die Zusatzversorgung im öffentlichen Dienst, NJ 2005, S. 199 ff.; Furtmayr/Wagner, Die Zusatzversorgung im öffentlichen Dienst - der heimliche Abschied von der Gesamtversorgung, NZS 2007, S. 299 ff.

13 Hierzu Gottwald (Fußn. 12), NJ 2005, S. 199, 202 ff.; auch Wegner-Wahnschaffe, Die Zusatzversorgung des öffentlichen Dienstes im Kontext veränderter Rahmenbedingungen ihrer Mitglieder, ZTR 2004, S. 395 ff.; Thiel, Entwicklungen und Tendenzen in der Zusatzversorgung des öffentlichen Dienstes, BetrAV 2005, S. 344 ff.

14 Furtmayr/Wagner (Fußn. 12), NZS 2007, S. 299, 302 ff.

Bestehende Anwartschaften sind zum Stichtag 31.12.2001 in Versorgungspunkte umgerechnet ( $\$ 18$ Abs. 2 BetrAVG) und als Startgutschrift in das Versorgungspunktesystem übertragen worden. Gegen diese Startgutschriften wurden gerichtliche Verfahren angestrengt. Zur Entscheidung des BGH vom 14.11.2007 (IV ZR 74/06), die sich zudem mit der Zulässigkeit der Systemumstellung befaßt, vgl. Hügelschäffer, Die Entscheidung des BGH zur Systemumstellung in der Zusatzversorgung des öffentlichen und kirchlichen Dienstes - Eine Zwischenbilanz, BetrAV 2008, S. 254 ff.; Wagner, Die Zusatzversorgung im öffentlichen Dienst - Schaffung einer verfassungskonformen Neuregelung der Satzung der VBL?, BetrAV 2008, S. 153 ff.; vgl. zur Rechtsprechung auch Wein, Aktuelle Rechtsprechung in der Zusatzversorgung des öffentlichen Dienstes, BetrAV 2007, S. 537 ff.

15 Battis, Bundesbeamtengesetz, 4. Aufl. 2009, § 4 Rdnr. 2; Lecheler, Der öffentliche Dienst, in: Isensee/Kirchhof (Hrsg.), Handbuch des Staatsrechts, Band III, 1988, § 72 Rdnr. 90.

Gemäß $§ 2$ Abs. 2 BRRG ist die Berufung in das Beamtenverhältnis nur zur Wahrnehmung hoheitsrechtlicher Aufgaben oder solcher Aufgaben, die aus Gründen der Sicherung des Staates oder des öffentlichen Lebens nicht ausschließlich Personen übertragen werden dürfen, die in einem privatrechtlichen Arbeitsverhältnis stehen, zulässig. Das Beamtenrechtsrahmengesetz ist mit Erlaß des Beamtenstatusgesetzes (BeamtStG) vom 17.6.2008 (BGBl. I S. 1010) weitestgehend aufgehoben worden. Siehe nunmehr $\S 3$ Abs. 2 BeamStG.

$16 \S 4$ BBG, § 3 Abs. 1 BeamtStG.

17 Die Funktion des Berufsbeamtentums besteht darin, gegründet auf Sachwissen, fachliche Leistung und loyale Pflichterfüllung eine stabile und gesetzestreue Verwaltung zu sichern. Vgl. BVerfGE 7, 155, 162; BVerfGE 56, 146, 162. 


\section{Verfassungsrechtlicher Hintergrund}

In Art. 33 Abs. 4 GG ist die institutionelle Garantie des Berufsbeamtentums niedergelegt. ${ }^{18}$ Entsprechend dieser Verfassungsnorm ist die Ausübung hoheitsrechtlicher Befugnisse als ständige Aufgabe in der Regel Angehörigen des öffentlichen Dienstes zu übertragen, die in einem öffentlich-rechtlichen Dienst- und Treueverhältnis stehen. Wesensmerkmale eines Dienst- und Treueverhältnisses sind insbesondere die gegenseitigen Treuepflichten; die Treuepflicht des Dienstherrn schließt dabei seine Fürsorge- und Schutzpflichten ein. 19

Die Regelung des öffentlichen Dienstes erfolgt nach Art. 33 Abs. 5 GG unter Berücksichtigung der hergebrachten Grundsätze des Berufsbeamtentums. Primäres Schutzgut des Art. 33 Abs. 5 GG ist die Existenz und die Funktionsfähigkeit der Institution des Berufsbeamtentums, die im politischen Kräftespiel eine stabile, gesetzestreue Verwaltung gewährleisten soll. ${ }^{20}$ In Ergänzung der in Art. 33 Abs. 4 GG niedergelegten institutionellen Garantie des Berufsbeamtentums enthält Art. 33 Abs. 5 GG zudem eine grundrechtsähnliche Individualgarantie: Jeder Beamte hat ein verfassungsbeschwerdefähiges subjektives Recht dahingehend, daß seine individuelle Rechtsstellung den hergebrachten Grundsätzen des Berufsbeamtentums entspricht. ${ }^{21}$

Mit Gesetz zur Änderung des Grundgesetzes vom 28.8.2006 22 ist in Art. 33 Abs. 5 GG das Gebot aufgenommen worden, das Recht des öffentlichen Dienstes „unter Berücksichtigung der hergebrachten Grundsätze des Berufsbeamtentums zu regeln und fortzuentwickeln." Die Neufassung hebt die Notwendigkeit einer Modernisierung und Anpassung des öffentlichen Dienstrechts an sich ändernde Rahmenbedingungen hervor. ${ }^{23}$ Ausweislich der Gesetzesbegründung bleibt die verfassungsrechtliche Garantie

18 Jachmann, in: v. Mangoldt/Klein/Starck (Hrsg.), Das Bonner Grundgesetz, 5. Aufl. 2005, Art. 33 Rdnr. 29; Battis, in: Sachs (Hrsg.), Grundgesetz Kommentar, 5. Aufl. 2009, Art. 33 Rdnr. 45; Masing, in: Dreier (Hrsg.), Grundgesetz Kommentar, 2. Aufl. 2006, § 33 Rdnr. 58.

19 Battis, in: Sachs (Hrsg.), Grundgesetz Kommentar (Fußn. 18), Art. 33 Rdnr. 51 ff.; Lecheler, Die „hergebrachten Grundsätze des Berufsbeamtentums“ in der Rechtsprechung des Bundesverfassungsgerichts und des Bundesverwaltungsgerichts, AöR 103 (1978), S. 349, 373.

20 BVerfGE 64, 367, 379; BVerfGE 56, 146, 162; Lecheler, in: Isensee/Kirchhof (Hrsg.), Handbuch des Staatsrechts (Fußn. 15), § 72 Rdnr. 65.

21 BVerfGE 8, 1, 17; BVerfGE 8, 28, 35; BVerfGE 12, 81, 87; Jachmann, in: v. Mangoldt/Klein/ Starck (Hrsg.), Das Bonner Grundgesetz (Fußn. 18), Art. 33 Rdnr. 40; Pieper, in: Schmidt-Bleibtreu/Hofmann/Hopfauf (Hrsg.), Grundgesetz Kommentar, 11. Aufl. 2008, Art. 33 Rdnr. 103; Battis, in: Sachs (Hrsg.), Grundgesetz Kommentar (Fußn. 18), Art. 33 Rdnr. 65.

22 BGBl. I S. 2034.

Hierzu u.a. Selmer, Die Föderalismusreform - Eine Modernisierung der bundesstaatlichen Ordnung?, JuS 2006, S. 1052 ff.

23 Solange nicht in den Kernbestand beamtenrechtlicher Strukturprinzipien eingegriffen, also keine strukturellen Veränderungen des Berufsbeamtentums vorgenommen werden, erlaubte das Grundgesetz bereits nach Art. 33 Abs. 5 a.F. eine stete Weiterentwicklung, mit dem Ziel, das Beamtenrecht veränderten Umständen anzupassen. Vgl. BVerfGE 43, 154, 168; BVerfGE 67, 1, 14; BVerfGE 97 , 
des Berufsbeamtentums unberührt, die hergebrachten Grundsätze des Berufsbeamtentums müssen weiterhin berücksichtigt werden. ${ }^{24}$ Fortzuentwickeln ist das Recht des öffentlichen Dienstes unter Beachtung der hergebrachten Grundsätze des Berufsbeamtentums als der hierbei anzuwendende Maßstab. ${ }^{25}$ Einfachgesetzliche Änderungen des Beamtenrechts, die mit den hergebrachten Grundsätzen des Berufsbeamtentums nicht in Einklang gebracht werden können, bleiben verfassungswidrig. ${ }^{26}$

\section{a) Hergebrachte Grundsätze des Berufsbeamtentums}

Die hergebrachten Grundsätze des Berufsbeamtentums definiert das Bundesverfassungsgericht in seiner ständigen Rechtsprechung als den Kernbestand von Strukturprinzipien, die allgemein oder doch ganz überwiegend während eines längeren, traditionsbildenden Zeitraums, mindestens unter der Reichsverfassung von Weimar, als verbindlich anerkannt und gewahrt worden sind. ${ }^{27} \mathrm{Zu}$ beachten ist, daß nicht jede Regelung des Beamtenrechts, die als hergebracht anzusehen ist, vom Schutzbereich des Art. 33 Abs. 5 GG erfaßt wird. Vielmehr sind nur diejenigen beamtenrechtlichen Regelungen vor einer Änderung geschützt, die das Bild des Berufsbeamtentums in seiner überkommenen Gestalt maßgeblich prägen, sodaß ihre Beseitigung auch das Wesen des Berufsbeamtentums antasten würde. ${ }^{28}$

Das Bundesverfassungsgericht hat in umfangreicher Rechtsprechung als hergebrachte Grundsätze des Berufsbeamtentums u.a. anerkannt:29

- Verpflichtung, wesentliche Inhalte des Beamtenverhältnisses durch Gesetz zu regeln

350, 376 f.; BVerfGE 117, 330, 348; Battis, Bundesbeamtengesetz (Fußn. 15), § 4 Rdnr. 11; Landau/Steinkühler, Zur Zukunft des Berufsbeamtentums in Deutschland, DVBl 2007, S. 133, 136.

24 BT-Drs. 16/813, S. 10.

25 BVerfGE 119, 247, 273; Kenntner, Aktuelle Rechtsprechung des Bundesverfassungsgerichts zum Beamtenrecht, JZ 2008, 340, 346 f.; Summer, Stabilisierung des Berufsbeamtentums durch die dritte Gewalt (Anmerkung zur Entscheidung des BVerfG's vom 19. September 2007 - 2 BvR 3/02 - ZBR 2007, 381), ZBR 2008, S. 158, 159.

26 Höfling/Burkiczak, Die Garantie der hergebrachten Grundsätze des Berufsbeamtentums unter Fortentwicklungsvorbehalt, Erste Überlegungen zur Änderung von Art. 33 Abs. 5 GG, DÖV 2007, S. 328, 333.

27 BVerfGE 8, 332, 343; BVerfGE 106, 225, 232; BVerfGE 117, 330, 344 f.; Isensee, Öffentlicher Dienst, in: Benda/Maihofer/Vogel (Hrsg.), Handbuch des Verfassungsrechts, 2. Aufl. 1994, § 32 Rdnr. 63; Günther, Die subjektivrechtliche Komponente des Funktionsvorbehaltes für das Berufsbeamtentum (Art. 33 Abs. 4, 5 GG), VerwArch 4/2008, S. 538, 544.

28 BVerfGE 43, 242, 278; BVerfGE 64, 323, 351; Maunz, in: Maunz/Dürig, Kommentar zum Grundgesetz, Bd. IV, Art. 33 Rdnr. 53; Kunig, in: v. Münch/Kunig (Hrsg.), Grundgesetz Kommentar, Bd. 2, 4./5. Aufl. 2001, Art. 33 Rdnr. 62.

29 Vgl. Maunz, in: Maunz/Dürig (Fußn. 28), Art. 33 Rdnr. 62 ff.; Battis, in: Sachs (Hrsg.), Grundgesetz Kommentar (Fußn. 19), Art. 33 Rdnr. 73 m.w.N.; Jachmann, in: v. Mangoldt/Klein/Starck (Hrsg.), Das Bonner Grundgesetz (Fußn. 18), Art. 33 Rdnr. 44 ff.; Lecheler (Fußn. 19), AöR 103 (1978), S. 349, $354 \mathrm{f}$. 
- Einstellung auf Lebenszeit und hauptberufliche Bindung30

- Recht auf amtsangemessene Amtsbezeichnung

- Laufbahnprinzip

- Leistungsprinzip

- Pflicht zur Amtsverschwiegenheit

- Parteipolitische Neutralität

- Haftungsprivileg

- Fürsorgepflicht des Dienstherrn

- Amtsangemessene Besoldung und Versorgung (Alimentationsprinzip)

- Gesetzesbindung der Besoldung und Versorgung

Die Beamtenversorgung betreffend ist das Alimentationsprinzip von Bedeutung. Neben dem Alimentationsprinzip prägen auch das Leistungsprinzip und das Lebenszeitprinzip als Grundprinzipien des Beamtenrechts das Recht der Beamtenversorgung. ${ }^{31}$

\section{b) Alimentationsprinzip}

Nach ständiger Rechtsprechung des Bundesverfassungsgerichts gehört das Alimentationsprinzip als wesentliche fürsorgerische Erscheinungsform des Beamtenrechts zu den hergebrachten Grundsätzen des Berufsbeamtentums, den der Gesetzgeber bei inhaltlicher Gestaltung des Beamtenrechts zu beachten hat. ${ }^{32}$ Das öffentlich-rechtliche Dienstund Treueverhältnis zwischen dem Beamten und seinem Dienstherrn ist auf Lebenszeit angelegt und verpflichtet den Beamten, seine ganze Arbeitskraft dem ihm übertragenen Amt zu widmen. ${ }^{33}$ Als Gegenleistung für die volle Hingabe für das Amt verpflichtet das Alimentationsprinzip den Dienstherrn, den Beamten und seine Familie ${ }^{34}$ lebenslang angemessen zu alimentieren und ihm nach seinem Dienstrang, nach der mit seinem Amt verbundenen Verantwortung und nach Maßgabe der Bedeutung des Berufsbeamtentums für die Allgemeinheit entsprechend der Entwicklung der allgemeinen wirtschaftlichen und finanziellen Verhältnisse und des allgemeinen Lebensstandards einen angemessenen Lebensunterhalt zu gewähren. ${ }^{35}$ Der Beamte muß stets über ein Nettoeinkommen verfügen, das seine rechtliche und wirtschaftliche Sicherheit sowie Unabhängigkeit ge-

30 Der Grundsatz der Hauptberuflichkeit beschränkt die Ausübung einer Nebentätigkeit. Vgl. Lecheler, in: Isensee/Kirchhof (Hrsg.), Handbuch des Staatsrechts (Fußn. 15), § 72 Rdnr. 52, 53; BVerfGE 55, 207, 240.

31 Ruland, in: Sachverständigenkommission Alterssicherungssysteme (Fußn. 9), Rdnr. 321 ff.; Battis, Beamtenversorgung, in: Cramer/Förster/Ruland (Hrsg.), Handbuch Altersvorsorgung, 1998, S. 117.

32 BVerfGE 8, 1, 16 f.; BVerfGE 44, 249, 265; BVerfGE 99, 300, 314.

33 BVerwGE 41, 316, 320.

34 So ist die Berücksichtigung der Kinderzahl bei der Beamtenbesoldung Teil der vom Dienstherrn geschuldeten Alimentation, da sichergestellt werden muß, daß der Beamte seine Unterhaltspflichten erfüllen kann. Vgl. BVerfGE 99, 300, 315.

35 BVerfGE 107, 218, 237; BVerfGE 114, 258, 287; Pieper, in: Schmidt-Bleibtreu/Hofmann/Hopfauf (Hrsg.), Grundgesetz Kommentar (Fußn. 21), Art. 33 Rdnr. 138. 
währleistet und ihm über die Befriedigung der Grundbedürfnisse hinaus einen seinem Amt angemessenen Lebenskomfort ermöglicht. ${ }^{36}$

Der vom Dienstherrn im Rahmen seiner Alimentationsverpflichtung geschuldete amtsangemessene Unterhalt wird durch das Leistungs- und Lebenszeitprinzip näher ausgefüllt. Als hergebrachter Grundsatz des Berufsbeamtentums beinhaltet das Leistungsprinzip insbesondere das Prinzip der Bestenauslese und ergänzt Art. 33 Abs. 2 $\mathrm{GG}^{37}$ durch eine auf den Schutz der ,erdienten Statusrechte“ ausgerichteten Komponente, um die Unabhängigkeit des Beamtentums und damit die Funktionsfähigkeit der Institution zu gewährleisten. ${ }^{38}$ Im Zusammenspiel mit dem Alimentationsprinzip hat auch das Lebenszeitprinzip die Aufgabe, zur Unabhängigkeit des Berufsbeamtentums im Interesse einer rechtsstaatlichen Verwaltung beizutragen, indem eine rechtliche und wirtschaftliche Sicherheit gewährleistet wird, um die Bereitschaft des Beamten zu einer an Gesetz und Recht orientierten, unparteiischen Amtsführung zu fördern. 39

Hervorzuheben ist, daß für die Versorgung des Beamten die Maßgeblichkeit des letzten Amtes festgeschrieben wird. ${ }^{40}$ Die Berechnung der Versorgungsbezüge auf Grundlage des zuletzt bezogenen Gehalts ist selbst ein hergebrachter Grundsatz des Berufsbeamtentums. ${ }^{41}$ Die Maßgeblichkeit des letzten Amtes bedeutet zum einen, daß ruhegehaltfähiges Einkommen die Einkünfte sind, die der Beamte aus dem von ihm vor seiner Versetzung in den Ruhestand bekleideten Amt bezogen hat. ${ }^{42}$ Denn die statusrechtliche Position, die der Beamte durch die Berufung in ein höheres Amt erlangt und mit der die fachliche Leistung, Eignung und Befähigung für das gegenüber dem bisherigen Amt

36 BVerfGE 117, 330, $351 \mathrm{f}$.

Die Amtsangemessenheit der Dienstbezüge des Beamten bemißt sich nach dem Nettoeinkommen. Vgl. BVerfGE 44, 249, 266; BVerfGE 81, 363, 376; BVerfGE 99, 300, 315.

37 Jeder Deutsche hat das Recht auf gleichen Zugang zu einem öffentlichen Amt nach Maßgabe seiner Eignung, Befähigung und fachlicher Leistung, Art. 33 Abs. 2 GG.

38 BVerfGE 64, 367, 385.

39 BVerfGE 70, 251, 267.

40 Vgl. § 5 Abs. 1 BVersG.

41 BVerfGE 11, 203, 210; BVerfGE 61, 43, 58; BVerfGE 76, 256, 324 f.; BVerwGE 24, 235, 243.

42 Die Beförderung eines Beamten ist bei den Versorgungsbezügen zu berücksichtigen. Die Mindestverweildauer im Beförderungsamt darf sich längstens auf zwei Jahre erstrecken: Das BVerfG hat mit Beschluß vom 20.3.2007 (2 BvL 11/04, BVerfGE 117, 372 ff.) in der Erstreckung der Wartefrist von zwei auf drei Jahre in $\S 5$ Abs. $3 \mathrm{~S}$. 1 BeamtVG einen Widerspruch gegen den nach Art. 33 Abs. 5 GG zu beachtenden Grundsatz der Versorgung aus dem letzten Amt gesehen. Gesichtspunkte, die im Gesetzgebungsverfahren vorgetragen wurden, wie die allgemeine Haushaltslage, Symmetrie von Dienst- und Versorgungszeiten sowie Änderungen im System der gesetzlichen Rentenversicherung, können, so das Bundesverfassungsgericht, die Erstreckung der Wartefrist auf drei Jahre nicht rechtfertigen. Hierzu Panzer, Die aktuelle Rechtsprechung des Bundesverfassungsgerichts zum öffentlichen Dienstrecht zwischen Bewahrung und Fortentwicklung, DÖV 2008, S. 707, 706 f.; Kenntner (Fußn. 25), JZ 2008, S. 340, 349 f.

Der Gesetzgeber hat auf die höchstrichterliche Rechtsprechung reagiert und die Wartefrist auf zwei Jahre herabgesetzt, Art. 4 Nr. 5 c Dienstrechtsneuordnungsgesetz (mit Wirkung ab 12.2.2009, Art. 17 Abs. 11 S. 1). 
höherwertige Amt anerkannt werden, muß sich wegen des engen Zusammenhangs zwischen Besoldung und Versorgung auf das Ruhegehalt auswirken. ${ }^{43}$ Zum anderen muß sich bei der Höhe der Versorgungsbezüge die Länge der aktiven Dienstzeit niederschlagen. ${ }^{44}$ Die Versorgung aus dem letzten Amt und die Abhängigkeit der Höhe der Versorgung von der Dauer der Dienstzeit sind Konsequenzen des Leistungs- und Lebenszeitprinzips: ${ }^{45}$ Der Beamte tritt aus seinem zuletzt innegehabten Amt in den Ruhestand, behält es unter Fortfall der Dienstleistungspflicht weiter; die abgeleistete Dienstzeit (ruhegehaltsfähige Dienstzeit) bestimmt als Zeitfaktor die Höhe des Versorgungsanspruchs bezogen auf das letzte Gehalt (ruhegehaltsfähige Dienstbezüge). ${ }^{46}$

Die unmittelbare Beziehung des Beamten zu seinem Dienstherrn im Rahmen des besonderen Dienst- und Treueverhältnisses bedingt, daß die Besoldung und Versorgung des Beamten unmittelbar durch den Dienstherrn erfolgen muß und nicht etwa auf einen personenverschiedenen Dritten übertragen werden darf. ${ }^{47}$ Die Versorgungslast liegt nach dem Alimentationsprinzip allein beim Dienstherrn, sodaß der Beamte auch nicht zur Entrichtung von Beiträgen zu seiner Altersvorsorge herangezogen wird. ${ }^{48}$

Es stellt sich die Frage, ob die Einführung einer Beitragspflicht für Beamte mit dem Alimentationsprinzip vereinbar wäre. ${ }^{49}$ Die Beantwortung dieser Frage hängt von der Einordnung der Beitragsfreiheit der Beamtenversorgung als hergebrachten Grundsatz des Berufsbeamtentums und somit als die Beamtenversorgung prägendes Prinzip ab. Wohl die überwiegende Ansicht sieht die Beitragsfreiheit als hergebrachten Grundsatz des Berufsbeamtentums an, mit der Folge, daß eine Verpflichtung der Beamten, ihre Altersvorsorge durch Beiträge mitzufinanzieren, für verfassungsrechtlich unzulässig erachtet wird. ${ }^{50}$ Aber auch die Literaturstimmen, die in der Einführung einer Beitragspflicht die Verpflichtung des Dienstherrn zur amtsangemessenen Alimentierung nicht

43 BVerfGE 56, 146, 163 f.; BVerfGE 61, 43, 58; BVerfGE 76, 256, 324.

44 BVerfGE 76, 256, 322; BVerfGE 114, 258, 286.

45 Ruland, Zur Zukunft von gesetzlicher Rentenversicherung und Beamtenversorgung, NVwZ 1995, S. $417,424$.

46 Fürst, Verfassungsrechtliche Grenzen einer Neuregelung der Beamtenversorgung, Verantwortung und Leistung, Heft 11, Juni 1984, S. 5, 8 f.

47 Petrasch, Die Alterssicherung der Beamten (Fußn. 11), S. 53; BVerfGE 76, 256, 319 f.

48 Battis, in: Alterssicherungssysteme im Vergleich, Schriften des WIÖD (Fußn. 8), S. 7; Merten, Aktuelle Probleme des Beamtenversorgungsrechts, NVwZ 1999, S. 809, 814.

49 Die Mehrheit der Sachverständigenkommission Alterssicherungssysteme, die durch Beschluß der Bundesregierung vom 1.6.1981 gebildet wurde, hatte sich für eine Beteiligung der Beamten an den Kosten ihrer Alterssicherung ausgesprochen. Vgl. Sachverständigenkommission Alterssicherungssysteme, Vergleich der Alterssicherungssysteme und Empfehlungen der Kommission, Gutachten der Sachverständigenkommission vom 19.11.1983, Berichtsband 1, S. 144 ff.

50 Merten (Fußn. 48), NVwZ 1999, S. 809, 814; Lecheler, in: Isensee/Kirchhof (Hrsg.), Handbuch des Staatsrechts (Fußn. 15), § 72 Rdnr. 55; BVerwGE 54, 177, 181 f.; offen gelassen BVerfG, 2 BvR 1673/03 vom 24.9.2007, Absatz-Nr. 55. 
tangiert sehen, ${ }^{51}$ verlangen, daß der Beamte innerhalb des öffentlich-rechtlichen Dienstund Treueverhältnisses rechtlich und wirtschaftlich abgesichert bleibt sowie seine personale Bindung zum Dienstherren für die Unterhaltsgewährung nicht geschmälert wird. ${ }^{52}$ Zudem darf die Einführung einer Beitragpflicht nicht dazu führen, daß die Versorgungsbezüge der Beamten auf Grundlage der erbrachten Beiträge und daher der im Lebensdurchschnitt erzielten Einkünfte bemessen werden. ${ }^{53}$ Die Bemessungsgrundlage der Versorgungsbezüge ist das vom Beamten vor der Versetzung in den Ruhestand zuletzt innegehabte Amt. Durch eine beitragsäquivalente Berechnung der Beamtenpensionen wird dieser prägende Grundsatz des Versorgungsrechts nicht gewahrt. 54

\section{Beurteilungsspielraum des Gesetzgebers bei der Gestaltung der Beamtenver- sorgung}

Der Gesetzgeber hat bei der Konkretisierung der aus Art. 33 Abs. 5 GG folgenden Verpflichtung zur amtsangemessenen Alimentierung einen weiten Gestaltungsspielraum. ${ }^{55}$ So besteht kein Anspruch des Beamten dahingehend, daß die für die Bemessung der Bezüge maßgeblichen Regelungen, unter denen er in das Beamten- und Ruhestandsverhältnis eingetreten ist, unverändert erhalten bleiben oder sich die Höhe der Bezüge nicht ändert. Der Gesetzgeber kann die Höhe der Versorgungsbezüge vielmehr kürzen. ${ }^{56}$ Doch können finanzielle Erwägungen und das Bemühen, Ausgaben zu sparen, für sich genommen nicht als ausreichende Legitimation für eine Kürzung der Altersversorgung angesehen werden. Nach der Rechtsprechung des Bundesverfassungsgerichts ist die vom Dienstherrn geschuldete Alimentierung keine dem Umfang nach beliebig variable Größe, die sich einfach nach den wirtschaftlichen Möglichkeiten der öffentlichen Hand, nach politischen Dringlichkeitsbewertungen oder nach dem Umfang der Bemühungen um die Verwirklichung des allgemeinen Sozialstaatsprinzips bemessen läßt. ${ }^{57}$ Daher müssen zu den finanziellen Erwägungen weitere Gründe hinzukom-

51 Battis/Kersten, Die Bildung von Versorgungsrücklagen für die Alterssicherung von Beamten - Zur Verfassungsmäßigkeit des § 14a BBesG, NVwZ 2000, S. 1337, 1339.

52 Ruland (Fußn. 45), NVwZ 1995, S. 417, 423.

53 Ruland, a.a.O.

54 Fürst, Verantwortung und Leistung Heft 11, Juni 1984 (Fußn. 46), S. 5, 13; Ruland, in: Sachverständigenkommission Alterssicherungssysteme (Fußn. 9), Rdnr. 367.

55 BVerfGE 76, 256, 295; BVerfGE 81, 363, 375 f.; BVerfGE 114, 258, 288; Pieper, in: SchmidtBleibtreu/Hofmann/Hopfauf (Hrsg.), Grundgesetz Kommentar (Fußn. 21), Art. 33 Rdnr. 117; Landau/Steinkühler (Fußn. 25), DVBl 2007, S. 133, 136.

56 BVerfGE 8, 1, 12 ff.; BVerfGE 18, 159, 166 f.; BVerfGE 70, 69, 79; Siebeck, Sozialversicherung und Beamtensicherung, Hergebrachte Grundsätze - Gegenwärtige Probleme, 1989, S. 41.

57 BVerfGE 44, 249, 264; BVerfGE 99, 300, 320; Zacher, Die Versorgung der Beamten, Richter und Soldaten, Insbesondere die Alters-, Dienstunfähigkeits- und Hinterbliebenenversorgung, 1984, S. 22. 
men, die im System der Altersversorgung liegen und die Kürzung von Bezügen als sachlich gerechtfertigt erscheinen lassen. ${ }^{58}$ Das Bundesverfassungsgericht verlangt hierbei, daß bei Absenkung des Versorgungsniveaus ein hinreichender Abstand zur Mindestversorgung des Beamten bestehen bleiben muß. Anderenfalls würde dies zu einer Nivellierung führen, die die Wertigkeit des Amtes nicht mehr hinreichend berücksichtigen würde. 59

\section{Einfachgesetzliche Regelung der Beamtenversorgung}

$\mathrm{Zu}$ den hergebrachten Grundsätzen des Berufsbeamtentums gehört die Verpflichtung, die wesentlichen Inhalte des Beamtenverhältnisses wie die Beamtenbesoldung und die Beamtenversorgung durch Gesetz zu regeln. 60

\section{Verteilung der Gesetzgebungskompetenzen zwischen Bund und Ländern}

Die wesentlichen Regelungskompetenzen zur Ausgestaltung der öffentlichrechtlichen Beamtenverhältnisse, sowohl die der Bundes- als auch die der Landes- und Kommunalbeamten, waren bis zum In-Kraft-Treten der sog. Föderalismusreform zum 1.9.2006 61 beim Bund konzentriert. Der Bund hatte die ausschließliche Gesetzgebungskompetenz zur Regelung der Rechtsverhältnisse der im Dienst des Bundes und der bundesunmittelbaren Körperschaften des öffentlichen Rechts stehenden Personen (Art. 73 Nr. 8 GG). Die Regelung der Besoldung und Versorgung der Beamten, die nicht als Bundesbeamten der ausschließlichen Gesetzgebungszuständigkeit des Bundes unterlagen, war dem Bund im Rahmen der konkurrierenden Gesetzgebung zugewiesen (Art. 74a GG). Der Bund hat seine Befugnisse durch Erlaß des Bundesbesoldungs- und Beamtenversorgungsgesetz weitgehend ausgeschöpft. Schließlich war die Regelung der Rechtsverhältnisse der im öffentlichen Dienste der Länder, Gemeinden und anderen Körperschaften des öffentlichen Rechts stehenden Personen der Rahmengesetzgebungskompetenz des Bundes zugewiesen (Art. 75 Abs. 1 S. 1 Nr. 1 GG). ${ }^{62}$

Die Föderalismusreform hat die Neuordnung der Regelungszuständigkeiten von Bund und Ländern zur Folge. ${ }^{63}$ Der Bund bleibt ausschließlich zuständig für die Rege-

58 BVerfGE 76, 256, 311; BVerfGE 114, 258, 291.

59 BVerfGE 114, 258, 295.

60 Hierzu Summer, Gedanken zum Gesetzesvorbehalt im Beamtenrecht, DÖV 2006, S. 249 ff.

61 Gesetz zur Änderung des Grundgesetzes vom 28.6.2006, BGB1. I S. 2034.

62 Der Bund hat auf Grundlage seiner Kompetenz das Rahmengesetz zur Vereinheitlichung des Beamtenrechts erlassen (Beamtenrechtsrahmengesetz, BRRG).

63 Vgl. hierzu u.a. Knopp/Schröder, Beamtenrechtliche und -politische Auswirkungen der Föderalismusreform, NJ 2007, S. $97 \mathrm{ff}$. 
lung der Rechtsverhältnisse der im Dienst des Bundes und der bundesunmittelbaren Körperschaften des öffentlichen Rechts stehenden Personen (Art. 73 Nr. 8 GG). Art. 74a GG wurde jedoch aufgehoben, mit der Folge, daß die Regelung der Besoldung und Versorgung der Landesbeamten und Landesrichter in die ausschließliche Gesetzgebungskompetenz der Länder fällt. ${ }^{64}$ Die bundesgesetzlichen Regelungen gelten fort, bis sie durch landesgesetzliche Regelungen vollständig abgelöst werden. An die Stelle der Rahmengesetzgebungskompetenz des Bundes zur Regelung der Rechtsverhältnisse der Landes- und Kommunalbediensteten ist die konkurrierende Gesetzgebungskompetenz getreten: Der Bund hat die Kompetenz zur Regelung der Statusrechte und -pflichten der Angehörigen des öffentlichen Dienstes der Länder, Gemeinden und anderen Körperschaften des öffentlichen Rechts, die in einem Dienst- und Treueverhältnis stehen, mit Ausnahme der Laufbahnen, Besoldung und Versorgung (Art. 74 Abs. 1 Nr. 27 GG). Am 17.6.2008 ist das Beamtenstatusgesetz ${ }^{65}$ zur einheitlichen Regelung des Statusrechts der Landes- und Kommunalbeamten verabschiedet worden, mit dessen Inkrafttreten zum 1.4.200966 das Beamtenrechtsrahmengesetz weitgehend aufgehoben wurde. ${ }^{67}$

\section{Reformen}

Die demographischen Entwicklungen, nämlich die sinkende Geburtenrate sowie die steigende Lebenserwartung und zunehmende Alterung der Gesellschaft werfen finanzielle Probleme für die im Umlageverfahren finanzierten Systeme sozialer Sicherung auf. Neben der gesetzlichen Rentenversicherung ist auch das aus dem laufenden Steueraufkommen finanzierte System der Beamtenversorgung betroffen. ${ }^{68}$ Die Notwendigkeit der langfristigen und nachhaltigen Sicherung hat in den letzten Jahren Reformen der

64 Beispielsweise hat der Bayerische Ministerrat am 26.1.2010 den Entwurf eines Gesetzes zum neuen Dienstrecht in Bayern beschlossen und das Gesetzespaket an den Bayerischen Landtag übermittelt. Gegenstand sind die vollständige Neuregelung des Laufbahn-, Besoldungs- und Versorgungsrecht wie die stufenweise Anhebung der Regelaltersgrenze auf 67 - und Änderungen im Beamtenstatusrecht. Bayern ist damit das erste Bundesland, das von den neuen Gesetzgebungskompetenzen im Dienstrecht umfassend Gebrauch macht (Bay. Staatskanzlei, Pressemitteilung vom 26.1.2010).

65 Gesetz zur Regelung des Statusrechts der Beamtinnen und Beamten in den Ländern (Beamtenstatusgesetz, BeamtStG) vom 17.6.2008, BGBl. I S. 1010. Das Gesetz legt, so die Gesetzesbegründung, die beamtenrechtlichen Grundstrukturen zur Gewährleistung der erforderlichen Einheitlichkeit des Dienstrechts fest und schafft durch klare Strukturen und den Abbau von bürokratischen Hemmnissen die Voraussetzungen für ein modernes und einheitliches Personalmanagement in der öffentlichen Verwaltung, vgl. BT-Drs. 16/4027, S. 1; BT-Drs. 16/7508 S. 1.

Hierzu u.a. Dillenburger, Das Beamtenstatusgesetz als neues Beamtenbundesrecht für Beamtinnen und Beamten der Länder, NJW 2009, S. 1115 ff.

$66 \S 25$ und $\S 50$ BeamtStG sind am Tag nach der Verkündung (20.6.2008) in Kraft getreten (§ 63).

67 Kapitel II und $\S 135$ des BRRG bleiben weiterhin in Kraft.

68 Ausführlich hierzu Dritter Versorgungsbericht der Bundesregierung, BT-Drs. 15/5821. 
Beamtenversorgung erforderlich gemacht. ${ }^{69}$ So wurden kostensenkende Reformmaßnahmen aus der gesetzlichen Rentenversicherung wirkungsgleich auf die Beamtenversorgung übertragen. ${ }^{70}$ Verfassungsrechtliche Bedenken gegen eine Orientierung an den Entwicklungen in der gesetzlichen Rentenversicherung bestehen dann nicht, wenn die Grundsätze, auf denen die Beamtenversorgung beruht, gewahrt werden. ${ }^{71}$ Das Alimentationsprinzip ist zwar als hergebrachter Grundsatz des Berufsbeamtentums verfassungsrechtlich dem Schutz des Art. 33 Abs. 5 GG unterworfen; solange aber die amtangemessene Versorgung gewährleistet wird, kann der Gesetzgeber auf gewandelte demographische, soziale und ökonomische Rahmenbedingungen reagieren. ${ }^{72}$

Die Reformen der letzten Jahre betreffend sind besonders hervorzuheben das Versorgungsreformgesetz vom 29.6.199873 und das Versorgungsänderungsgesetz vom 20.12.200174. Zuletzt hat die Beamtenversorgung mit dem Dienstrechtsneuordnungsgesetz Änderungen erfahren. ${ }^{75}$

\section{a) Versorgungsreformgesetz 1998}

Mit dem Versorgungsreformgesetz vom 29.6.1998 wurde u.a. § 14 a in das Bundesbesoldungsgesetz eingefügt. Diese Vorschrift schreibt in ihrem Absatz 1 vor, daß beim Bund und bei den Ländern Versorgungsrücklagen als Sondervermögen aus der Minderung der Besoldungs- und Versorgungsanpassungen gebildet werden ${ }^{76}$, um die Versorgungsleistungen angesichts der demographischen Veränderungen und des Anstiegs der Zahl der Versorgungsempfänger sicherzustellen. Das Besoldungs- und Versorgungsniveau soll in gleichmäßigen Schritten von durchschnittlich 0,2\% abgesenkt werden (§ 14

69 Siehe Färber, Mittel- und langfristige Entwicklung der Beamtenversorgung, in: Cramer/Förster/Ruland (Hrsg.), Handbuch Altersvorsorgung (Fußn. 31), S. 973 ff.

70 Hierzu Färber/Funke/Walther, Die Entwicklung der Beamtenversorgung in Deutschland seit 1992 und künftige Finanzierungsprobleme der Gebietskörperschaften, DÖV 2009, S. 133, 135 ff.

71 BVerfGE 114, 258 ff.; Lenze, Wie sicher sind verfassungsrechtlich die Pensionen?, NVwZ 2006, S. 1129, 1232.

72 Färber/Funke/Walther (Fußn. 70), DÖV 2009, S. 133, 139.

73 Gesetz zur Umsetzung des Versorgungsberichts (Versorgungsreformgesetz 1998 - VReformG), BGB1. I S. 1666. Hierzu Meier, Das Versorgungsreformgesetz 1998 - Die gesetzgeberische Umsetzung des Versorgungsberichts der Bundesregierung, NVwZ 1998, S. 1246 ff.; Battis, Das Versorgungsreformgesetz 1998, NJW 1998, S. 2653 ff.

74 BGBl. I S. 3926. Hierzu Ruland, Noch einmal davongekommen - Zur Reform der Beamtenversorgung, NJW 2002, S. 948 ff.

75 Gesetz zur Neuordnung und Modernisierung des Bundesdienstrechts vom 5.2.2009, BGB1. I 160. Hierzu Ziekow, Die Fortentwicklung des Dienstrechts der Bundesbeamten, DÖV 2008, S. 569 ff.

76 Die Minderung der Besoldungs- und Versorgungsanpassungen sind nicht als echte Beiträge der Beamten zu ihrer Versorgung zu qualifizieren, sondern lediglich als Kürzungen ihres Gehalts. Vgl. Battis, Bundesbeamtengesetz (Fußn. 15), Einleitung Rdnr. 24; BVerwGE 117, 305, 311. 
a Abs. 1 S. 2 BBesG). ${ }^{77}$ Das aus den Versorgungsrücklagen gebildete Sondervermögen ist zweckgebunden und nur zur Verwendung künftiger Versorgungsausgaben zu verwenden ( $\S 14$ a Abs. 2 S. 3 BBesG). Den Bund betreffend wird das Sondervermögen durch das Bundesministerium des Innern verwaltet. Die Verwaltung der Mittel des Sondervermögens obliegt der Deutschen Bundesbank. Für die Anlage der Mittel gelten besondere Regeln. ${ }^{78}$

Die Bildung von Versorgungsrücklagen hat nicht zur Folge, daß der Dienstherr seine eigene Verpflichtung zur amtsangemessenen Alimentation des Ruhestandsbeamten und seiner Hinterbliebenen auf einen „Dritten“ überträgt. Denn das zweckgebundene Sondervermögen ist lediglich eine Ansammlung von Kapital. Rechtsbeziehungen zwischen dem Beamten und dem Sondervermögen werden nicht begründet; die personale Bindung zwischen dem Beamten und seinem Dienstherrn besteht ohne Modifizierung von Pflichten und Rechten fort. ${ }^{79}$ In diesem Zusammenhang ist auszuführen, daß zur Finanzierung der Altersvorsorgekosten für Bundesbeamte und Soldaten neben dem Sondervermögen „Versorgungsrücklage des Bundes“ mit Wirkung ab dem 1.1.2007 ein Sondervermögen „Versorgungsfonds des Bundes“ errichtet und somit eine kapitalgedeckte Altersversorgung als weitere Säule zur Finanzierung der Beamtenversorgung eingeführt wurde. Für Einstellungen ab dem 31.12.2006 ist der jeweilige Dienstherr verpflichtet, durch regelmäßige Zuweisungen, deren Höhe sich laufbahnabhängig auf Grundlage versicherungsmathematischer Berechnungen nach Prozentsätzen der jeweiligen ruhegehaltsfähigen Dienstbezüge ergibt, Rücklagen zu bilden. ${ }^{80}$ Einzelheiten werden in einer Rechtsverordnung geregelt. ${ }^{81}$ Die Mittel des Versorgungsfonds, die aus Steuern aufzubringen sind, werden von der Deutschen Bundesbank verwaltet. Erstattungen aus dem Versorgungsfonds für die Versorgungsaufwendungen des einbezogenen Personenkreises erfolgen erst ab dem Jahr 2020. Dadurch soll ein ausreichendes Anwachsen des Fondsvermögens sichergestellt werden. 82

77 Hierzu Färber, in: Cramer/Förster/Ruland (Hrsg.), Handbuch Altersvorsorgung (Fußn. 69), S. 973, $985 \mathrm{f}$.

78 Vgl. § 5 VersRücklG.

Im Freistaat Bayern z.B. verwaltet das Staatsministerium der Finanzen das zweckgebundene Sondervermögen, Art. 5 BayVersRücklG.

79 BVerwGE 117, 305, $310 \mathrm{f}$.

80 Zweckgebundene Versorgungsfonds gibt es auch in einigen Bundesländern. Im Freistaat Bayern z.B. ist der Versorgungsfonds mit Wirkung zum 1.1.2008 für Neueinstellungen ab dem 31.12.2007 errichtet worden. Vgl. Art. 13 ff. BayVersRücklG.

81 Vgl. Versorgungsfondszuweisungsverordnung vom 11.4.2007, BGB1. I S. 549.

82 Vgl. $\S 13$ ff. VersRücklG. 


\section{b) Versorgungsänderungsgesetz 2001}

Die Einführung einer Regelung zur Bildung einer Versorgungsrücklage war die Reaktion auf die mit Rentenreformgesetz 1999 vom 16.12.199783 geänderte und um einen demographischen Faktor ergänzte Rentenformel der gesetzlichen Rentenversicherung. Durch den demographischen Faktor sollten die jährlichen Rentenanpassungen verringert, in der Folge das Rentenniveau abgesenkt werden. ${ }^{84}$ Doch wurde der demographische Faktor wieder aus der Rentenformel entfernt. ${ }^{85}$ Die Rentenreform 2001 führte sodann im Wesentlichen zur Änderung der Rentenanpassungsformel und die Förderung der privaten Altersvorsorge der Versicherten durch Gewährung von steuerlichen Vergünstigungen und Zuschüssen. 86

Im Kontext zur Rentenreform 2001 wurden mit dem Versorgungsänderungsgesetz vom 20.12.2001 u.a. die Senkung des Versorgungsniveaus ${ }^{87}$ und gleichzeitig die steuerliche Förderung der privaten Altersvorsorge ${ }^{88}$ in die Beamtenversorgung eingeführt. Zugleich wurde die Bildung der Versorgungsrücklage nach $\S 14$ a BBesG bis 2011 ausgesetzt und bis $2017^{89}$ verlängert. Zudem wurden aktive Beamte in die gesetzlich geförderte, steuerbegünstigte private kapitalgedeckte Altersvorsorge einbezogen. ${ }^{90}$

Das BVerfG hat die Regelung in $\S 14$ a BBesG ${ }^{91}$ und die Absenkung des Versorgungsniveaus ${ }^{92}$ nicht beanstandet und im Wesentlichen ausgeführt: Änderungen in der

83 BGBl. I S. 2998.

84 BT-Drs. 13/8011, S. 48 f.

85 Gesetz zu Korrekturen in der Sozialversicherung und zur Sicherung der Arbeitnehmerrechte vom 19.12.1998, BGBl. I S. 3843.

86 Gesetz zur Ergänzung des Gesetzes zur Reform der gesetzlichen Rentenversicherung und zur Förderung eines kapitalgedeckten Altersvorsorgevermögens (Altersvermögens-Ergänzungsgesetz - AVmEG) vom 21.3.2001, BGB1. I 403; Gesetz zur Reform der gesetzlichen Rentenversicherung und zur Förderung eines kapitalgedeckten Altersvorsorgevermögens (Altersvermögensgesetz - AVmG) vom 26.6.2001, BGB1. I S. 1310. Siehe auch BT-Drs. 14/9503, S. 26 ff.

Hierzu u.a. Kreikebohm, in: ders. (Hrsg.), Gesetzliche Rentenversicherung Kommentar, 3. Aufl. 2008, Einleitung Rdnr. $47 \mathrm{ff}$.

87 Der Höchstversorgungssatz sinkt von $75 \%$ der ruhegehaltsfähigen Bezüge bei 40 Dienstjahren auf $71,75 \%$ (1,79375 \% für jedes Jahr ruhegehaltfähiger Dienstzeit anstatt 1,875 \%).

88 Mit der Folge, daß Beiträge für eine zusätzliche Altersvorsorge als Sonderausgaben steuerlich absetzbar sind (vgl. § 10 a EStG).

89 Das mit den Rücklagen gebildete Sondervermögen sollte ursprünglich bereits ab 2014 zur Finanzierung der Versorgungslasten eingesetzt werden.

90 Siehe Darstellung bei Färber/Funke/Walther (Fußn. 70), DÖV 2009, S. 133, 137.

91 BVerfG, 2 BvR 1673/03 vom 24.9.2007, NVwZ 2008, S. 195 ff.

Gegenstand der Entscheidung waren die Bundesbesoldungs- und Bundesversorgungsanpassungsgesetze 1999 und 2000, soweit diese in Anlehnung an $\S 14$ a BBesG Verminderungen der Besoldungsund Versorgungsanpassungen von jeweils $0,2 \%$ zum 1.6.1999, 1.1.2001 und 1.1.2002 vorsahen.

92 BVerfGE 114, 258 ff.; hierzu Lenze (Fußn. 71), NVwZ 2006, S. 1129 ff.; Hase, Rentenversicherung und Beamtenversorgung, die Versorgungsreform 2001 und das Urteil des Bundesverfassungsgerichts vom 27. September 2005, in: Becker/Kaufmann/v. Maydell/Schmähl/Zacher (Hrsg.), Alterssicherung in Deutschland, Festschrift für Franz Ruland zum 65. Geburtstag, 2007, S. 496, 501 ff. 
gesetzlichen Rentenversicherung und die diesen zugrundeliegenden Entwicklungen können Anlaß bieten, diese in der Beamtenversorgung systemkonform nachzuführen. Dabei beruht die Berücksichtigungsfähigkeit von Einschnitten in die Alterseinkünfte der Rentner auf der herausragenden Bedeutung der Einkommen der privatrechtlich beschäftigten Arbeitnehmer für die verfassungsrechtlich gebotene Alimentierung. Doch können das System der gesetzlichen Rentenversicherung und dessen Änderungen nur dann zur Bestimmung der Angemessenheit der Versorgungsbezüge und zur Rechtfertigung deren Absenkung herangezogen werden, wenn diese mit den strukturellen Unterschieden der beiden Versorgungssysteme vereinbar sind. Führen Änderungen in der gesetzlichen Rentenversicherung dazu, daß eine angemessene Alterssicherung nur durch zusätzliche private Altersvorsorge gesichert ist, dann ist eine Übertragbarkeit auf das Versorgungsrecht ausgeschlossen. ${ }^{93}$ Denn der Gesetzgeber muß die Bifunktionalität der Beamtenversorgung beachten. D.h. zwischen dem Leistungsniveau der gesetzlichen Rentenversicherung und dem Leistungsniveau der Beamtenversorgung muß eine deutliche Spanne liegen; ansonsten würde der Doppelcharakter der Beamtenversorgung als Verbundsystem aus qualifizierter Grundsicherung und zusätzlicher betrieblicher Altersversorgung verkannt werden. ${ }^{94}$

\section{c) Neuordnung des Dienstrechts}

Das Dienstrechtsneuordnungsgesetz vom 5.2.200995 nimmt bei der Novellierung des Beamtenversorgungsgesetzes in erster Linie die wirkungsgleiche Übertragung von Maßnahmen in der gesetzlichen Rentenversicherung unter Berücksichtigung der Unterschiedlichkeit der Alterssicherungssysteme vor. Dies geschieht u.a. durch - im Zusammenhang mit der stufenweise Anhebung des Pensionseintrittsalters im Bundesbeamtengesetz ${ }^{96}$ wie in der gesetzlichen Rentenversicherung 97 - rentengleiche Versorgungsregelungen bei schrittweise Anhebung des Pensionseintrittsalters auf das 67. Lebensjahr,

Das BVerfG stellte u.a. fest, daß ein hergebrachter Grundsatz des Berufsbeamtentums dahingehend, daß der Höchstversorgungssatz $75 \%$ der ruhegehaltsfähigen Bezüge betragen muß, nicht existiert. $\mathrm{Da}$ die Absenkung des Versorgungsniveaus auch Beamte betraf, die vor dem 1.1.2002 in den Ruhestand versetzt wurden, wurde nicht als unzulässige echte Rückwirkung (= Rückwirkung in Form einer Rückbewirkung von Rechtsfolgen) und auch nicht als Verstoß gegen den Grundsatz des Vertrauensschutzes gewertet. Denn das Ruhegehalt von Beamten steht unter dem Vorbehalt der Abänderbarkeit. Vgl. auch BVerfGE 70, 69, 91.

93 BVerfGE 114, 258, 292 f., 295.

94 Ruland, in: Sachverständigenkommission Alterssicherungssysteme (Fußn. 9), Rdnr. 381.

95 Gesetz zur Neuordnung und Modernisierung des Bundesdienstrechts, BGBl. I S. 160. Hierzu Battis, Das Dienstrechtsneuordnungsgesetz, NVwZ 2009, S. 409 ff.

96 Bundesbeamtengesetz vom 5. Februar 2009, BGB1. I S. 160.

97 Das Renteneintrittsalter in der gesetzlichen Rentenversicherung wird von 2012 an bis 2029 stufenweise von derzeit 65 auf 67 erhöht. Vgl. Gesetz zur Anpassung der Regelaltersgrenze an die demographische Entwicklung und zur Stärkung der Finanzierungsgrundlagen der gesetzlichen Rentenversicherung (RV-Altersgrenzenanpassungsgesetz) vom 20.4.2007, BGBl. I S. 554. 
insbesondere mit der Einführung eines abschlagsfreien Pensionseintritts nach 45 Jahren und Beibehaltung der bisherigen Antragsaltersgrenze von 63 Jahren bei einer schrittweisen Erhöhung des maximalen Versorgungsabschlags auf $14,4 \%$ bei vorzeitigem Pensionseintritt auf Antrag. 98

\section{Gesetzliche Bestimmung des Ruhegehalts}

Das bundesrechtliche Beamtenversorgungsgesetz regelt die Arten der Versorgung für den erfaßten Personenkreis ( $\S 1,2$ BeamtVG) sowie die Voraussetzungen und den Umfang der jeweiligen Versorgungsbezüge. 99

\section{a) Anspruch auf Ruhegehalt und Höhe des Ruhegehalts}

Mit dem Ende des Monats, in dem der Beamte auf Lebenszeit das 67. Lebensjahr vollendet, tritt er im Allgemeinen in den Ruhestand. ${ }^{100}$ Die bisher normierte Regelaltersgrenze von 65 Jahren wird bei Personen, die ab dem 31.12.1946 geboren wurden stufenweise angehoben; bei Personen, die vor dem 1.1.1947 geboren sind, bleibt sie bestehen (vgl. § $51 \mathrm{BBG}$ ).

Der Beamte erlangt einen Anspruch auf das Ruhegehalt, das auf der Grundlage der ruhegehaltfähigen Dienstbezüge und der ruhegehaltfähigen Dienstzeit berechnet wird, nach Ableistung einer Dienstzeit von mindestens fünf Jahren mit Eintritt in den Ruhestand. Wurde der Beamte wegen Dienstunfähigkeit in den Ruhestand versetzt und hat er seine Dienstunfähigkeit nicht durch grobes Verschulden herbeigeführt, dann bedarf es der Erfüllung der Wartezeit von fünf Jahren nicht ( 44 BeamtVG).

Die Höhe des Ruhegehalts ist in $§ 14$ BeamtVG geregelt. Sie ist aus der Multiplikation der ruhegehaltsfähigen Dienstzeit mit dem Steigerungssatz von 1,79375 \% und mit den ruhegehaltsfähigen Dienstbezügen zu ermitteln:

ruhegehaltsfähige Dienstzeit (Zeitfaktor) x Steigerungssatz (Wertfaktor) x ruhegehaltsfähige Dienstbezüge $=$ Ruhegehalt

Die Summe aus ruhegehaltsfähiger Dienstzeit und dem Steigerungssatz wird als Ruhegehaltssatz bezeichnet. 101 Der Ruhegehaltssatz ist auf 71,75\% (= 40 Dienstjahre) begrenzt.

98 BT-Drs. 16/7076, S. 3. Siehe Färber/Funke/Walther (Fußn. 70), DÖV 2009, S. 133, 138.

99 Anzumerken ist, daß das BeamtVG auch die Unfallfürsorge für Beamte regelt ( $§ 30 \mathrm{ff}$.). Soweit die beamtenrechtlichen Unfallfürsorgevorschriften greifen, sind Beamte in der gesetzlichen Unfallversicherung versicherungsfrei ( $\$ 4$ Abs. 1 Nr. 1 SGB VII).

Landesrecht wird im Folgenden nicht behandelt.

100 Für bestimmte Personenkreise gelten Sonderaltersgrenzen. Die vorzeitige Versetzung in den Ruhestand wird nicht behandelt.

101 Lümmen/Grunefeld/Kempf, Beamtenversorgung, 1. Aufl. 2003, § 14, S. 66. 
Die Regelung in $\S 14$ BeamtVG in seiner heutigen Fassung mit Wirkung ab 1.1.2003 wurde mit dem Versorgungsänderungsgesetz vom 20.12.2001 eingeführt und ist daher in Verbindung mit der Übergangsregelung in $\S 69$ e BeamtVG zu lesen: 102 Der Steigerungssatz von 1,79375\% findet erst nach der achten auf den 31.12.2002 folgenden allgemeinen Anpassung der Versorgungsbezüge nach § 70 BeamtVG ${ }^{103}$ Anwendung. Für Versorgungsfälle vor diesem Zeitpunkt gilt $\S 14$ BeamtVG in seiner alten Fassung, d.h. als Steigerungssatz sind 1,875\% zugrunde zu legen; der Ruhegehaltssatz ist auf $75 \%$ (= 40 Dienstjahre) der ruhegehaltsfähigen Bezüge begrenzt. Es gilt jedoch die Besonderheit, daß ab der ersten auf den 31.12.2002 folgenden Anpassung der Versorgungsbezüge nach $\S 70$ BeamtVG bis zur siebten Anpassung durch einen sich schrittweise verändernden Anpassungsfaktor, der gesetzlich vorgegeben ist, die Anpassung der ruhegehaltsfähigen Dienstbezüge gesenkt wird. ${ }^{104}$ Nach der achten auf den 31.12.2002 folgenden Versorgungsanpassung wird schließlich der Ruhegehaltssatz selbst abgesenkt. Mit dieser Übergangsregelung erfolgt eine schrittweise Absenkung des Höchstversorgungssatzes von $75 \%$ auf $71,75 \%$ und des jährlichen Steigerungssatzes von 1,875\% auf $1,79375 \%$.

In der gesetzlichen Rentenversicherung beruht die Berechnung des Monatsbetrags der Rente auf der sog. Rentenformel, die lautet: 105

persönliche Entgeltpunkte (§ 66 SGB VI) x Rentenartfaktor (§ 67 SGB VI) x aktueller Rentenwert ( $§ 68$ SGB VI) ${ }^{106}$

\section{b) Mindestversorgung}

Beamte erhalten mindestens $35 \%$ ihrer ruhegehaltsfähigen Dienstbezüge als Ruhegehalt. An die Stelle des auf diese Weise festgesetzten Ruhegehalts treten $65 \%$ der ru-

102 Die Anwendung des $\S 69$ e BeamtVG hat zur Folge, daß sich die Besoldungs- und Versorgungsbezüge der Beamten unterschiedlich entwickeln werden. Doch existiert kein Grundsatz dahingehend, daß der Gesetzgeber verpflichtet wäre, bei Anpassungen der Bezüge eine strikte Parallelität zwischen Besoldung und Versorgung zu gewährleisten. Vgl. BVerfGE 114, 258, 281.

$103 \S 70$ Abs. 1 BeamtVG: „Werden die Dienstbezüge der Besoldungsberechtigten allgemein erhöht oder vermindert, sind von demselben Zeitpunkt an die Versorgungsbezüge durch Bundesgesetz entsprechend zu regeln.“

Das BVerfG hat hierzu festgestellt, daß in der in $\S 70$ Abs. 1 BeamtVG niedergelegten Verpflichtung zur Anpassung der Versorgungsbezüge an Veränderungen der Besoldungsbezüge bedingt durch die allgemeine Entwicklung der wirtschaftlichen und finanziellen Verhältnisse (§ 14 Abs. 1 BBesG) eine Konkretisierung des Alimentationsprinzips zu sehen ist (BVerfGE 114, 258, 288 f.).

104 Hier handelt es sich um einen technischen Vorgang; die ruhegehaltsfähigen Dienstbezüge werden mit einem sich bei jeder Anpassung nach $\S 70$ BeamtVG verändernden Faktor multipliziert. Vgl. Lümmen/Grunefeld/Kempf, Beamtenversorgung (Fußn. 101), § 69 e, S. 192.

105 Hierzu u.a. Michaelis, Rentenberechnung, Rentenzahlung, Rentenanpassung, in: Ruland (Hrsg.), Handbuch der gesetzlichen Rentenversicherung (Fußn. 6), S. 695 ff.

106 Der aktuelle Rentenwert verändert sich zum 1. Juli eines jeden Jahres und beträgt seit dem 1. Juli 2009 27,20 EUR (West) / 24,13 EUR (Ost). 
hegehaltsfähigen Dienstbezüge aus der Endstufe der Besoldungsgruppe A 4 dann, wenn dies günstiger ist ( $§ 14$ Abs. 4 BeamtVG). Das Institut der Mindestversorgung ist eine Konsequenz der Verpflichtung des Dienstherrn zur lebenslangen Alimentation des Beamten und seiner Familie. Die Mindestversorgung wirkt als versorgungsrechtliches Existenzminimum, wenn die berechneten Ruhestandsbezüge eine amtsangemessene Alimentation nicht gewährleisten können; beispielsweise, wenn die ruhegehaltsfähigen Dienstzeiten und Dienstgehälter eine der beruflichen Stellung des Beamten entsprechende Versorgung nicht sicherstellen können. 107

Eine Mindestrente kennt die gesetzliche Rentenversicherung im Vergleich nicht. ${ }^{108}$

\section{c) Besteuerung von Versorgungsbezügen}

Versorgungsbezüge unterliegen der Einkommenssteuer. Renten aus der gesetzlichen Rentenversicherung und aus Zusatzversorgungen dagegen sind nur mit ihrem Ertragsanteil einkommenssteuerpflichtig. ${ }^{109}$ Die ungleiche Behandlung von Beamtenpensionen und Renten hat das BVerfG zuletzt ${ }^{110}$ mit seiner Entscheidung am 6.3.2002 beanstandet und den Gleichheitssatz (Art. 3 Abs. 1 GG) als verletzt angesehen. 111

$\mathrm{Ab} 2005$ sieht nunmehr das Alterseinkünftegesetz vom 5.7.2004112 einen schrittweisen Übergang zur nachgelagerten Besteuerung vor. Nach Ablauf der Übergangsphase werden Beamtenpensionen und Renten ab 2040 steuerrechtlich gleich behandelt. ${ }^{113}$

107 Lümmen/Grunefeld/Kempf, Beamtenversorgung (Fußn. 101), § 14, S. 71.

$108 \mathrm{Zu}$ ergänzenden Leistungen der sozialhilferechtlichen Grundsicherung im Alter und bei Erwerbsminderung siehe $\S \S 41$ ff. SGB XII. Zum 31.12.2006 betrug die Zahl der Empfänger von Grundsicherungsleistungen im Alter 365.000, Statistisches Bundesamt, Statistisches Jahrbuch 2008, S. 217. Hinzuweisen ist für die gesetzliche Rentenversicherung auf $\S 262$ SGB VI, der Mindestentgeltpunkte für Beitragszeiten mit vollwertigen Pflichtbeiträgen für Versicherungszeiten vor dem 1.1.1992 vorsieht. Betroffen sind Versicherte, die mindestens 35 Jahre mit rentenrechtlichen Zeiten aufweisen und im Verlauf ihres Erwerbslebens Einkünfte unter dem Durchschnittseinkommen aller Versicherten erzielt haben.

109 Vgl. $\S 19$ Abs. 1 S. 1 Nr. 2, 22 Nr. 1 S. 3 a EStG.

110 Vgl. bereits BVerfGE 54, 11, 34.

111 BVerfGE 105, $135 \mathrm{ff}$.

Ausführlich hierzu Liesenfeld, Die Besteuerung von Renten und Pensionen - Anmerkungen zum Urteil des BVerfG vom 6.3.2002 aus verfassungsrechtlicher Sicht, DStR 2002, S. 1834.

112 Gesetz zur Neuordnung der einkommenssteuerrechtlichen Behandlung von Altersvorsorgeaufwendungen und Altersbezügen, BGBl. I S. 1427.

113 Hierzu Birk/Wensmann, Die Besteuerung der Aufwendungen für die Altersvorsorge und der Alterseinkommen, in: Ruland/Rürup (Hrsg.), Alterssicherung und Besteuerung (Fußn. 1), § 9 Rdnr. 9 ff; Ruland, in: von Maydell/Ruland/Becker (Hrsg.), Sozialrechtshandbuch (Fußn. 8), § 17 Rdnr. 222. 


\section{Bewertung}

Das öffentlich-rechtliche Dienst- und Treueverhältnis zwischen dem Beamten und seinem Dienstherrn wird durch die hergebrachten Grundsätze des Berufsbeamtentums ausgestaltet. Der verfassungsrechtliche Sonderstatus der Beamten bedingt die gesonderte Absicherung im Alter durch die Beamtenversorgung, die der Fürsorgepflicht des Dienstherrn entspringt, als in das Beamtenrecht integriertes Sondersystem sozialer Sicherheit neben der gesetzlichen Rentenversicherung. ${ }^{114}$

Als hergebrachter Grundsatz des Berufsbeamtentums steht das Alimentationsprinzip im Vordergrund, das die amtsangemessene Versorgung des Beamten und seiner Familie anordnet. Bei der Konkretisierung der Alimentierungsverpflichtung steht dem Gesetzgeber ein weiter Gestaltungsspielraum zu, der durch die tragenden Strukturprinzipien des Berufsbeamtentums beschränkt wird. So ist das Ruhegehalt auf Grundlage der ruhegehaltsfähigen Dienstbezüge im zuletzt innegehabten Amt zu berechnen. Des Weiteren muß sich die Länge der aktiven Dienstzeit in der Höhe der Versorgungsbezüge niederschlagen. Die Finanzierung der Beamtenversorgung erfolgt schließlich allein durch den Dienstherrn.

Ausgehend von Reformen der gesetzlichen Rentenversicherung ${ }^{115}$ kann die Änderung der Beamtenversorgung nur unter Beachtung der strukturellen Unterschiede beider Versorgungssysteme erfolgen.

114 Die Beamtenversorgung wird von der in Art. 33 Abs. 5 GG verankerten institutionellen Garantie des Berufsbeamtentums erfaßt. Siehe Ruland (Fußn. 45), NVwZ 1995, S. 417, 422.

$115 \mathrm{Zu}$ Reformdiskussionen vgl. Ruland, in: von Maydell/Ruland/Becker (Hrsg.), Sozialrechtshandbuch (Fußn. 8), § 17 Rdnr. 231 ff. 


\title{
Die Alterssicherung der Beamten in Frankreich
}

\author{
Otto Kaufmann
}

I. Staat und Verwaltung

1. Politische und verfassungsrechtliche Rahmenbedingungen

2. Der Aufbau des öffentlichen Dienstes
a) Der öffentliche Dienst des Staates
b) Der öffentliche Dienst der Gebietskörperschaften
c) Der öffentliche Dienst des Krankenhauswesens

II. Die Grundstruktur des Beamtentums

1. Allgemeines

2. Das Beamtenstatut

3. Die Prinzipien des Beamtentums

4. Die Laufbahn
a) Eintritt in den Dienst
b) Die Vergütung
c) Die Stellung des Beamten
d) Beendigung der Laufbahn

III. Die Alterssicherung

1. Nebeneinander verschiedener Sicherungssysteme

a) Das allgemeine System

b) Die Sondersysteme

2. Die Basisalterssicherung im öffentlichen Dienst

a) Die Alterssicherung der Beamten

aa) Angleichung an das allgemeine Alterssicherungssystem

bb) Die Versicherten

cc) Die Voraussetzungen zum Bezug der Leistungen

dd) Die Höhe der Leistungen

b) Leistungen an Hinterbliebene

3. Freiwillige und obligatorische Zusatzversicherung für den öffentlichen Dienst

4. Die Finanzierung und Besteuerung

IV. Zusammenfassende Bemerkungen: Angleichung der Alterssicherungssysteme 